\title{
Treatment of Colovaginal Fistula with Coaxial Placement of Covered and Uncovered Stents
}

In the colon self-expanding stents may be used for palliation of malignant obstruction as well as being employed as a temporary measure prior to definitive surgery [1]. Difficulties include a high rate of migration and stent occlusion. Successful treatment of enteric fistulas has been described [2-5]. We present a case of a large colovaginal fistula that was successfully sealed at the second attempt by coaxial placement of an uncovered and a covered stent. A 78-year-old lady was admitted with a history of altered bowel habit, abdominal pain and increasing vaginal discharge. Computed tomography showed a sigmoid carcinoma arising within diverticular disease, confirmed on sigmoidoscopy. She was unfit for surgery and was referred for colonic stenting. By this time she had free faeculent vaginal discharge and was completely bed-bound. A large sigmoido-vaginal fistula within a sigmoid stricture was demonstrated on fluoroscopy (Figure 1). The stricture was traversed with a 7-Fr angiographic catheter and hydrophilic wire. After exchange for a stiff wire, a 17-cm, 18-mm covered oesophageal nitinol stent (Choo; DiagMed, UK) was deployed (Figure 2). Injection of contrast showed no residual leak. From the following day the patient passed stools normally and the vaginal discharge stop-

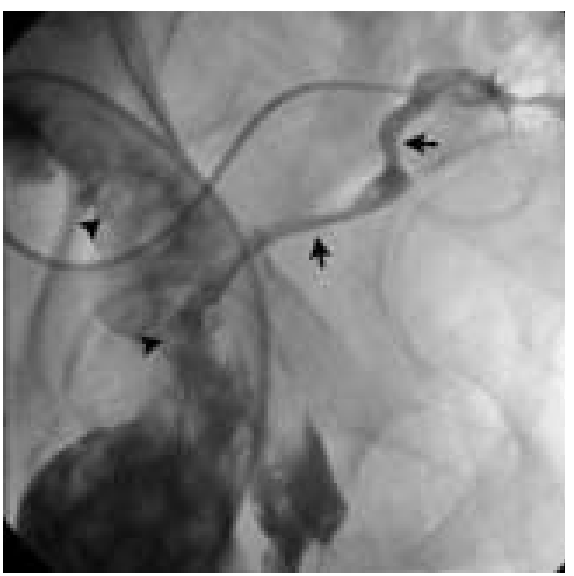

Figure 1 Injection of contrast through an angiographic catheter into the sigmoid stricture shows free drainage through a large fistula (arrows) into the vagina. A separate catheter for air insufflation is present (arrowheads). ped completely. At 7 days later the patient passed the stent into the toilet and developed recurrent intestinal obstruction and vaginal discharge. A second attempt was made at stenting. An uncovered 9-cm, 22mm Enteral Wallstent (Boston Scientific UK) was deployed as an anchor, through which a further Choo stent was placed coaxially (Figure $\mathbf{3}$ ). The patient made an uneventful recovery, with bowel motions returning to normal and complete resolution of vaginal discharge. The stents were still in situ and functioning 6 months later and the fistula was sealed. Relieving colonic obstruction with an uncovered stent and reducing the pressure proximal to this may be sufficient to allow a fistula to close. In difficult cases a combination with a covered stent should be considered.

\section{H.-U. Laasch, L. Wilbraham, A. Marriott, D. F. Martin}

Academic Department of Gastrointestinal Radiology, South Manchester University Hospitals NHS Trust, Manchester, and University of Central Lancashire, UK.

\section{References}

${ }^{1}$ Baron TH, Dean PA, Yates, 3rd MR et al. Expandable metal stents for the treatment of colonic obstruction: techniques and outcomes. Gastrointest Endosc 1998; 47: 277-286

${ }^{2}$ Grunshaw ND, Ball CS. Palliative treatment of an enterorectal fistula with a covered metallic stent. Cardiovasc Intervent Radiol 2001; 24: 438 -440

${ }^{3}$ Cwikiel W, Andren-Sandberg A. Malignant stricture with colovesical fistula: stent insertion in the colon. Radiology 1993; 186: 563 - 564

${ }^{4}$ Jeyarajah AR, Shepherd JH, Fairclough PDet al. Effective palliation of a colovaginal fistula using a self-expanding metal stent. Gastrointest Endosc 1997; 46: $367-369$

${ }^{5}$ Kyoto Y, Iwasaki Y, Kaji T et al. Gastrointestinal fistulas: treatment with covered stents. Abdom Imaging 2001; 26: 570-573

\section{Corresponding Author}

\section{H.-U. Laasch, MD}

Academic Department of Gastrointestinal Radiology, Acute Block, South Manchester University Hospitals

Southmoor Road, Wythenshawe, Manchester M23 9LT, UK

Fax: $\quad$ +44-161-2916201

E-mail: hul@smtr.nhs.uk
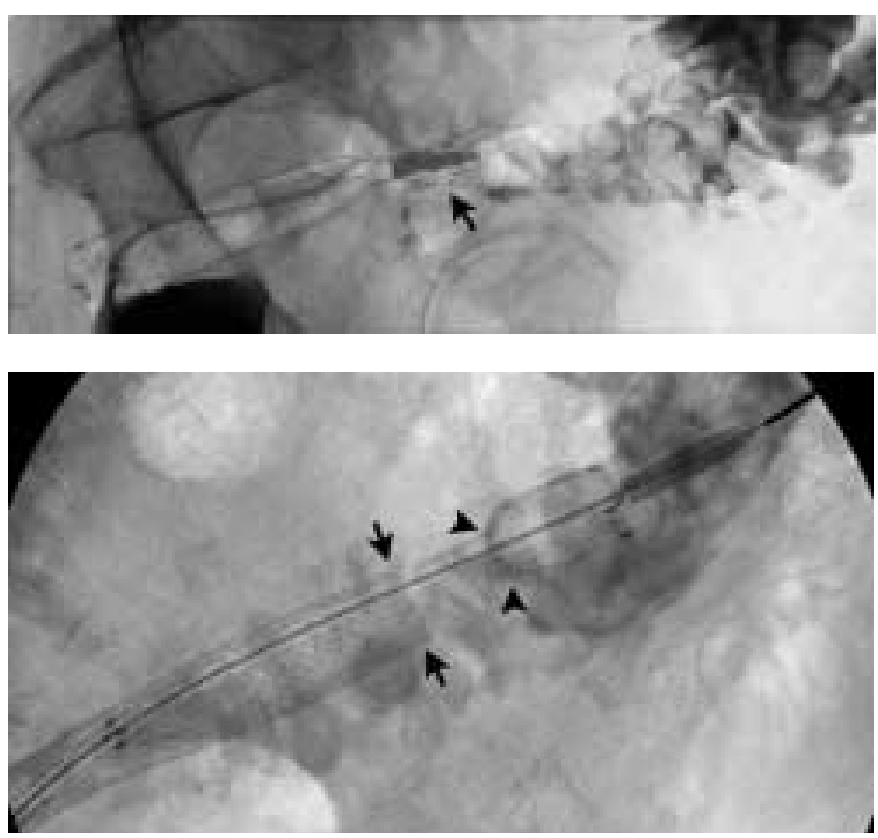

Figure 2 A 17-cm nitinol stent is inserted over a wire and positioned with the middle marker (arrow) at the level of the fistula. This migrated after 1 week.

Figure 3 Coaxial placement of a Choo stent. The distal end is deployed (arrowheads) beyond the Enteral Wallstent (arrows). 